\title{
Differential outcomes in triple negative breast cancer observed among the major ethnic groups in Singapore
}

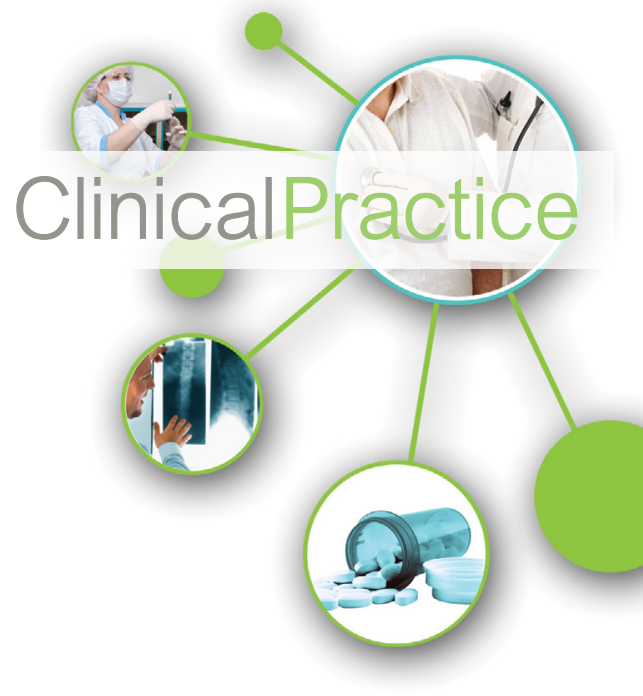

\begin{abstract}
Objectives: Although Triple Negative Breast Cancers (TNBCs) are known as a particularly aggressive subtype, some women have a favorable prognosis and recurrence after the initial few years is uncommon. In this study, we reviewed the outcome of women with TNBC and evaluated factors potentially useful for risk stratification.

Methods: Retrospective review was performed of 345 patients diagnosed with TNBC at two local institutes from 2006 to 2011. Detailed analyses focused on 315 women without metastasis.

Results: TNBC accounted for $11.3 \%$ of cancers diagnosed and was most prevalent among Indian women. Disease recurrence was a significant determinant of overall survival $(P<0.001)$ and apart from the disease stage $(P<0.001)$, it was independently associated with ethnicity $(\mathrm{P}=0.011)$. Recurrence was two-fold higher among Malay and Indian women compared to Chinese women $(P=0.027)$ and 5-year recurrence-free survival was significantly shorter compared to Chinese women ( $P=0.031$, HR 2.575, 95\% Cl 0.165 to $0.915 ; P=0.034, H R 2.090,95 \% \mathrm{Cl} 0.514$ to 0.929 ). On the other hand, 5 -year recurrence-free survival was similar between Malay and Indian women ( $\mathrm{P}=0.987)$. This survival difference could not be attributed to differences in disease factors or stage at presentation, and apart from more Malay women receiving radiation $(\mathrm{P}=0.020)$, due to higher rates of breast conservation in this group, the women all received similar treatments.
\end{abstract}

Conclusions: Ethnicity and disease stage were identified as independent predictors of disease recurrence in women with TNBC, with Chinese women having better survival outcomes.

Keywords: Triple-negative breast cancer, ethnicity, recurrence

\section{Objective}

Triple Negative Breast Cancers (TNBC), tumors negative for estrogen receptor (ER), progesterone receptor (PR) and human epidermal growth factor receptor (HER)2 , are known as a more aggressive subtype often associated with a poor outcome. The propensity for early systemic relapse and early nodal spread suggest an inherent tendency for metastasis and higher rates of intrinsic resistance to conventional chemotherapy $[1,2]$. Although TNBC accounts for only $10 \%$ to $15 \%$ of all breast cancers, they present a treatment dilemma. High recurrence rates coupled with the absence of targeted therapies have led to a low threshold for chemotherapy and many women with small node-negative cancers are recommended chemotherapy. In spite of this, systemic failure remains a problem and distant relapse rates are well above that observed with the other subtypes. Nevertheless, the disease follows a much more favorable course in some women. Those who remain disease-free after the initial 2 to 3 years are unlikely to recur thereafter and in fact, relapse rates fall below that seen with ER-positive tumors beyond 5 years [1,3,4]. It would thus appear that women with TNBC can be stratified into a high-risk group, characterized by early systemic failure and poor survival, and a low-risk group with long-term event-free survival.

The variation in outcomes can be attributed to the molecular heterogeneity within TNBC, which is in fact merely a collective term used to describe tumors that do not express ER, PR, and HER2 receptors. Molecular characterization has identified six distinct subtypes from among TNBCs [5]. These subtypes differ with regards to gene profiles and account for the differential response to treatments and outcomes. For
Serene Ee Ling Tang ${ }^{1}$, Aric Wei Zheng Lee ${ }^{1,2}$, Evan Yu Ze Teo ${ }^{1}$, Sherwin Kuah', Hui Miao ${ }^{3}$, Patrick Chan ${ }^{1}$, Mikael Hartman ${ }^{3,4}$, and Ern Yu Tan ${ }^{1^{*}}$

${ }^{1}$ Department of General Surgery, Tan Tock Seng Hospital, Singapore ${ }^{2}$ Lee Kong Chian School of Medicine, Singapore

${ }^{3}$ Saw Swee Hock School of Public Health, National University of

Singapore, Singapore

${ }^{4}$ Department of General Surgery, National University Hospital, Singapore

*Author for correspondence:

Ern_Yu_Tan@ttsh.com.sg 
instance, TNBCs of the basal BL1 and BL2 subtypes are highly chemo-sensitive and frequently achieve a complete pathological response, while those of the mesenchymal subtype have a less favorable outcome and are particularly predisposed to early relapse [5]. This would explain the apparent paradox of frequent complete pathological response, suggesting chemo-sensitivity, yet high rates of systemic failure, implying chemo-resistance instead. By stratifying TNBCs by molecular profiles, subtyping offers better prognostication than what is possible with traditional indicators and aids decision making by providing a more accurate estimate of chemotherapy benefit. Furthermore, subtyping can identify potential candidates for the clinical testing of novel therapeutic agents, many of which are highly specific targeted agents, thereby making the accurate definition of target subgroups essential. Despite these clear benefits, molecular profiling has remained outside of routine clinical practice because of the high costs. This has also hindered large-scale clinical validation to evaluate how molecular profiles can guide treatment decisionmaking. There is, therefore, a need to re-evaluate currently available clinicopathological indicators and to identify those that could potentially serve as surrogate predictors of outcomes. Better risk stratification would facilitate further studies to explore how treatments can be tailored to specific subgroups, and could also help guide the development of molecular assays to stratify TNBCs. In this study, we reviewed women diagnosed with TNBC and evaluated the clinicopathological parameters that could potentially stratify these women according to 5-year overall and recurrence-free survival.

\section{Materials and Methods}

A retrospective review was performed of 345 women diagnosed and treated for TNBC at two institutes in Singapore (Tan Tock Seng Hospital, TTSH, and the National University Hospital, NUH) from between $1^{\text {st }}$ January 2006 to $31^{\text {st }}$ December 2011. There were 217 women in the TTSH cohort and 128 women in the NUH cohort. This study has Ethics Committee approval, which was obtained from the National Healthcare Group DomainSpecific Review Board (Study approval code: DSRB2013/00597). Women diagnosed prior to 2006 when HER2 testing was not yet routine, male patients and those who did not receive treatment at either institute after the initial diagnosis were excluded. Detailed analyses were performed by the 315 women who presented with non-metastatic disease. All patients were followed up for at least 5 years; median followup period was 62.1 months (ranging from 2.1 to 119.3 months) and 160 of the 315 patients with the non-metastatic disease were followed up for more than 5 years.

Tumour ER, PR and HER2 status was determined by immunohistochemistry according to standardized laboratory protocols at the respective institutes. At TTSH, staining protocols and thresholds for ER and PR were revised in 2010 after the ASCO/CAP update. Prior to this, formalin fixed paraffin embedded tumor sections were stained with the anti-ER antibody (Neomarker MS750) and anti-PR antibody (Dako M3569) and tumors were considered positive if $10 \%$ or more tumor cells stained with at least moderate intensity. After 2010, sections were stained with the anti-ER antibody (Novocastra NCL-ER-6F11) and anti-PR antibody (Ventana 1E2) and considered positive if at least $1 \%$ of tumor cells stained positive [6]. The anti-HER2 antibody (Neomarker MS-441) was in use in 2006 and tumors were considered positive if at least $10 \%$ of tumor cells exhibited intense membranous staining and equivocal if at least $10 \%$ of tumor cells exhibited moderate membranous staining; tumors not fulfilling either these criteria were considered negative. In 2007, the anti-HER2 antibody (Neomarker RM-9103) was used instead and tumors were considered positive if more than $30 \%$ of tumor cells exhibited uniform intense membrane staining and equivocal if at least $10 \%$ of tumor cells exhibited complete circumferential membrane staining that was non-uniform or weak in intensity, or if $30 \%$ or fewer tumor cells exhibited intense complete membrane staining [7]. At NUH, the same IHC protocols and thresholds were in use for the entire study period; antibodies used were that of anti-ER antibody (Ventana SP1 clone), anti-PR antibody (Ventana 1E2 clone) and antiHER2 antibody (Ventana 4B5 clone). Tumors were considered ER/PR positive if at least $1 \%$ of tumor cells stained positive. Thresholds for HER2 status were similar to those in use at TTSH. At both institutes, tumors with an equivocal IHC result were subjected to Fluorescent in-situ Hybridization (FISH) and all 
assays were performed at NUH. Amplification of the HER2/neu gene was detected using Vysis PathVysion HER2 DNA Probe kit and a HER2 gene signal to chromosome 17 signal ratio of greater than or equal to 2.0 was considered positive.

Both institutes followed the same guidelines and treatments carried out were similar. Surgery was performed according to standard techniques. Modified radical mastectomy involved the removal of the entire breast together with an ellipse of overlying skin and the nipple areolar complex but preserving both pectoralis muscles. Patients were given the choice of immediate breast reconstruction if they were deemed, suitable candidates. In Wide Local Excision (WLE), the tumor was resected together with a margin of normal breast tissue and a surgical margin of at least $1 \mathrm{~mm}$ was considered adequate. Sentinel lymph node biopsy was performed at the same setting unless the nodes were clinically palpable or if there was already pre-operative histological confirmation of nodal metastasis. Full axillary nodal dissection was performed in such cases, and in all cases where the sentinel node was positive for metastatic disease. Whole breast irradiation was routinely offered to all women after WLE. A total dose of 50Gy was administrated in fractions of $2 \mathrm{~Gy}$ over a period of 6 weeks. In 2010, a hyperfractionated regimen (42.5Gy in 16 fractions) was also offered, mostly to women with node-negative disease [8]. Post-mastectomy radiation was recommended to women with tumors larger than $5 \mathrm{~cm}$, pre-menopausal women with nodal involvement and to all women with $\mathrm{N} 2$ (4 or more nodes) involvement. The benefit of radiation was discussed on a case-by-case basis in post-menopausal women with $\mathrm{N} 1$ disease. An additional boost of 10Gy to the tumor bed was routinely given, and a higher dose of $16 \mathrm{~Gy}$ was considered if there were concerns about residual disease post-resection. A 2-field technique was used post-WLE for node-negative disease and a 3-field technique, to include the whole breast or chest wall, the level I and II nodes and supraclavicular fossa, was applied when there was a nodal disease. Systemic treatment recommendations were in accordance with existing NCCN guidelines and first-line regimens comprising anthracyclines and taxanes were the most often used. Chemotherapy was recommended for all node-positive disease and for node-negative tumors that were staged as T1c or larger, in most instances. The benefit of chemotherapy was discussed on a case-by-case basis in women with node-negative T1b tumors.

Data was collected from the clinical records and included demographic data, mode of clinical presentation, treatments received, standard pathological and outcome parameters. Clinical outcome was correlated with patient demographics and standard clinicopathological parameters and comparisons were made between women from the three major ethnic groups. Univariate analyses with the chi-squared test, chi-squared test for trend and one-way ANOVA were used as appropriate and were performed with GraphPadPrism version 6 (GraphPad Software Inc., San Diego CA). Cox proportional hazards model was used to identify independent predictors of 5-year overall and recurrencefree survival and was performed with the Stata package release 11.0 (Stata Corporation, 4905 Lakeway Drive, College Station, Texas 77845, USA). A full model was first created to include all potentially important explanatory variables. At each step, the variable with the smallest contribution to the model was removed, until a final backward stepwise model was obtained. Kaplan-Meier survival curves of disease-free survival were calculated using GraphPad Prism as well. A 2-tailed $\mathrm{P}$ value test was used in all analyses and a $\mathrm{P}$ value of less than 0.05 was considered statistically significant.

\section{Results}

A total of 345 women with TNBC were collectively diagnosed at both institutes and this accounted for $11.3 \%$ (345 of 3064) of all breast cancers diagnosed during the 6-year period. Prevalence was highest among Indian women (14.5\%), followed by Malays (9.1\%) and was lowest among Chinese women (7.5\%). Details are presented in TABLE 1. Most women were diagnosed with early Stage I and II cancers and 30 women presented with de novo metastatic disease. All 315 women with non-metastatic disease underwent surgery; 181 were treated with mastectomy and 134 with Wide Local Excision (WLE). Seventy percent (223 of 315) of these women (with the nonmetastatic disease) received chemotherapy; most were given as adjuvant treatment. Patient and tumor characteristics were similar between the two institutes, as were the types of treatments 


\begin{tabular}{|c|c|c|c|}
\hline Parameter & Total Number (\%) & TTSH Number (\%) & NUH Number (\%) \\
\hline \multirow{2}{*}{ Median age (years) } & $345(100)$ & $204(100)$ & $141(100)$ \\
\hline & $54(25-90)$ & $55(30-90)$ & $51(25-83)$ \\
\hline $\begin{array}{l}\text { Ethnicity } \\
\text { Chinese } \\
\text { Malay } \\
\text { Indians } \\
\text { Others }^{*}\end{array}$ & $\begin{array}{l}229(66.4) \\
45(13.0) \\
36(10.4) \\
35(10.1)\end{array}$ & $\begin{array}{l}143(70.1) \\
23(11.3) \\
22(10.8) \\
16(7.8)\end{array}$ & $\begin{array}{l}86(61.0) \\
22(15.6) \\
14(9.9) \\
19(13.5)\end{array}$ \\
\hline $\begin{array}{l}\text { Disease Stage } \\
\text { I } \\
\text { II } \\
\text { III } \\
\text { IV }\end{array}$ & $\begin{array}{l}94(27.2) \\
147(42.6) \\
74(21.4) \\
30(8.7)\end{array}$ & $\begin{array}{l}55(26.9) \\
80(39.2) \\
51(25.0) \\
18(8.8)\end{array}$ & $\begin{array}{l}39(27.7) \\
67(47.5) \\
23(16.3) \\
12(8.5)\end{array}$ \\
\hline Median tumour size $(\mathrm{mm})$ & $25(1-170)$ & $25(1-110)$ & $25(6-170)$ \\
\hline \begin{tabular}{|l|} 
Nodal status \\
N0 \\
N1 \\
N2 \\
N3 \\
Unknown \\
\end{tabular} & $\begin{array}{l}185(53.6) \\
75(21.7) \\
23(6.7) \\
39(11.3) \\
23(6.7) \\
\end{array}$ & $\begin{array}{l}104(51.0) \\
46(22.5) \\
17(8.3) \\
27(13.2) \\
10(4.9)\end{array}$ & $\begin{array}{l}81(57.4) \\
29(20.6) \\
6(4.3) \\
12(8.5) \\
13(9.2) \\
\end{array}$ \\
\hline \begin{tabular}{|l|} 
Tumour grade \\
1 \\
2 \\
3 \\
Unknown \\
\end{tabular} & $\begin{array}{l}7(2.0) \\
54(15.6) \\
253(73.3) \\
31(9.0) \\
\end{array}$ & \begin{tabular}{|l}
$6(2.9)$ \\
$30(14.7)$ \\
$142(69.3)$ \\
$26(12.7)$ \\
\end{tabular} & \begin{tabular}{|l}
$1(0.7)$ \\
$24(17.0)$ \\
$111(78.7)$ \\
$5(3.5)$ \\
\end{tabular} \\
\hline $\begin{array}{l}\text { Tumour histology } \\
\text { IDC } \\
\text { ILC } \\
\text { Metaplastic carcinoma } \\
\text { Medullary carcinoma } \\
\text { Mucinous carcinoma } \\
\text { Papillary carcinoma } \\
\text { Tubular carcinoma }\end{array}$ & $\begin{array}{l}319(92.5) \\
8(2.3) \\
10(2.9) \\
3(0.9) \\
2(0.6) \\
2(0.6) \\
1(0.3)\end{array}$ & $\begin{array}{l}182(89.2) \\
7(3.4) \\
7(3.4) \\
3(1.5) \\
2(1.0) \\
2(1.0) \\
1(0.5)\end{array}$ & $\begin{array}{l}137(97.2) \\
1(0.7) \\
3(2.1) \\
0 \\
0 \\
0 \\
0\end{array}$ \\
\hline $\begin{array}{l}\text { Surgery received } \\
\text { Mastectomy } \\
\text { Wide local excision } \\
\text { No surgery } \\
\end{array}$ & $\begin{array}{l}181(52.5) \\
134(38.8) \\
30(9.3)\end{array}$ & $\begin{array}{l}110(53.9) \\
70(34.3) \\
18(8.8) \\
\end{array}$ & \begin{tabular}{|l|}
$71(50.4)$ \\
$64(45.4)$ \\
$12(8.5)$ \\
\end{tabular} \\
\hline $\begin{array}{l}\text { Chemotherapy } \\
\text { Neoadjuvant } \\
\text { Adjuvant } \\
\text { Palliative } \\
\text { None } \\
\text { Unknown } \\
\end{array}$ & $\begin{array}{l}40(11.6) \\
183(53.0) \\
21(6.1) \\
90(26.1) \\
11(3.2) \\
\end{array}$ & $\begin{array}{l}22(10.8) \\
108(52.9) \\
12(5.9) \\
51(25.0) \\
11(5.4) \\
\end{array}$ & $\begin{array}{l}18(12.8) \\
75(53.2) \\
9(6.4) \\
37(26.2) \\
2(1.4) \\
\end{array}$ \\
\hline $\begin{array}{l}\text { Radiation therapy } \\
\text { Neoadjuvant } \\
\text { Adjuvant } \\
\text { Palliative } \\
\text { None } \\
\text { Unknown } \\
\end{array}$ & $\begin{array}{l}1(0.3) \\
177(51.3) \\
9(2.6) \\
149(43.2) \\
9(2.6) \\
\end{array}$ & $\begin{array}{l}1(0.5) \\
105(51.5) \\
4(2.0) \\
87(42.6) \\
7(3.4) \\
\end{array}$ & $\begin{array}{l}0(0) \\
72(51.1) \\
5(3.5) \\
62(44.0) \\
2(1.4) \\
\end{array}$ \\
\hline \begin{tabular}{|l} 
Recurrence \\
Locoregional \\
Distant (systemic) \\
\end{tabular} & $\begin{array}{l}63(18.3) \\
26(7.5) \\
55(15.9) \\
\end{array}$ & \begin{tabular}{|l}
$38(18.6)$ \\
$20(9.8)$ \\
$34(16.7)$ \\
\end{tabular} & \begin{tabular}{|l}
$25(17.7)$ \\
$6(4.3)$ \\
$21(14.9)$ \\
\end{tabular} \\
\hline Death & $78(22.6)$ & $50(24.5)$ & $28(19.9)$ \\
\hline
\end{tabular}

received. Over the follow-up period, there were 78 deaths, with 52 occurring within the first 5 years. Of these, 17 deaths were possibly unrelated to breast cancer, having occurred in women who had not developed disease recurrence or treatment-related complications. Disease recurrence developed in 63 women, and the majority of these recurrences (55 of 63, 87.3\%) were systemic in nature. Distant metastasis was the first event in the majority of women, who had presented with worrisome symptoms. The asymptomatic systemic disease was found during re-staging work-up in 4 women who had presented with locoregional recurrence.
Locoregional recurrence was the first event in 20 women and followed the detection of systemic disease in another 6 women. The locoregional recurrence remained an isolated event in only 8 women, and systemic disease later developed in the other 12 women. Median recurrence-free survival was 59.5 months (ranging from 3.2 to 119.3 months).

Overall survival was significantly poorer among women with TNBC compared to women with tumors of other subtypes ( $\mathrm{P}<0.001$, OR 2.123, 95\% CI 1.739 to 4.010$)$ (data not shown). Overall survival showed a 
strong association with the occurrence of disease recurrence $(\mathrm{P}<0.001$, HR 13.113, 95\% CI 6.104 to 28.169) (TABLE 2), but did not seem affected by the treatments received. There was no significant association with either chemotherapy or radiation treatments, and although univariate analysis suggested a survival benefit in favor of women treated with WLE $(\mathrm{P}=0.002$, HR $0.316,95 \mathrm{CI} 0.152$ to 0.659 ), this association was no longer observed after adjusting for age at diagnosis, disease stage and tumor grade $(\mathrm{P}=0.288)$ (TABLE 2).

We next evaluated factors associated with disease recurrence. Recurrence, especially if systemic, mostly occurred within the first 5 years. Seven women developed locoregional recurrence after 5 years and another woman was found with systemic disease 60.2 months after surgery. Disease stage was an independent predictor of disease recurrence $(\mathrm{P}<0.001, \mathrm{HR}$ $5.435,95 \%$ CI 2.110 to 14.000 ), with the risk, increased 5-fold in women presenting with Stage III disease (TABLE 3). There was, again, no significant association with the treatments received; the association with WLE was no longer significant in the multivariate analysis $(\mathrm{P}=0.370)$. Twenty-one of the 134 women
(15.6\%) treated with WLE subsequently defaulted whole breast radiation and 3 later developed ipsilateral breast recurrence without systemic disease. Another 14 women defaulted post-mastectomy radiation and 5 later developed distant recurrence despite having completed chemotherapy. Chemotherapy was recommended to 292 of 315 (93\%) women and only 33 women declined it. Only 23 women, were not recommended chemotherapy; 9 had node-negative T1a tumors, 8 were older than 75 years of age and 2 were deemed not fit for chemotherapy (no clear documentation were available for the remaining 4). Of the 56 women who did not receive chemotherapy, 3 relapsed locally and another 12 (7 with Stage II disease and 5 with Stage III disease) relapsed systemically. Overall, chemotherapy did not appear to significantly reduce the risk of recurrence $(\mathrm{P}=0.558)$.

Interestingly, an association with ethnicity was observed. Disease recurrence was 2.8fold higher in Indian women $(\mathrm{P}=0.011$, HR 2.761, 95\% CI 1.263 to 6.039$)$ and 1.5 fold higher in Malay women $(\mathrm{P}=0.245$, HR $1.55395 \%$ CI 0.739 to 3.263) compared to Chinese women; the former reaching statistical

TABLE 2. Univariate and multivariate Cox proportional hazards model analyses of overall survival correlated with clinicopathological parameters and treatments received in patients with non-metastatic disease.

\begin{tabular}{|c|c|c|c|c|}
\hline \multirow[b]{2}{*}{ Parameter } & \multicolumn{2}{|l|}{ Univariate analysis } & \multicolumn{2}{|l|}{ Multivariate analysis } \\
\hline & $\mathrm{HR}(95 \% \mathrm{Cl})$ & $P$ value & HR (95\% Cl) & $P$ value \\
\hline Median age (years) & $1.007(0.984-1.031)$ & 0.546 & $1.011(0.981-1.043)$ & 0.480 \\
\hline $\begin{array}{l}\text { Ethnicity } \\
\text { Chinese } \\
\text { Malay } \\
\text { Indians }\end{array}$ & $\begin{array}{l}1.000 \text { [Reference] } \\
1.919(0.920-4.003) \\
0.972(0.380-2.484)\end{array}$ & $\begin{array}{l}0.082 \\
0.952\end{array}$ & $\begin{array}{l}1.000 \\
1.766(0.728-4.285) \\
0.791(0.269-2.329)\end{array}$ & $\begin{array}{l}0.209 \\
0.670\end{array}$ \\
\hline $\begin{array}{l}\text { Disease stage } \\
\text { I } \\
\text { II } \\
\text { III }\end{array}$ & $\begin{array}{l}1.000 \text { [Reference] } \\
3.667(1.263-10.643) \\
9.072(3.147-26.158)\end{array}$ & $\begin{array}{l}0.017 \\
<0.001\end{array}$ & $\begin{array}{l}1.000 \text { [Reference] } \\
2.006(0.623-6.459) \\
2.968(0.838-10.514)\end{array}$ & $\begin{array}{l}0.244 \\
0.092\end{array}$ \\
\hline $\begin{array}{l}\text { Tumour grade } \\
1 \\
2 \\
3 \\
\end{array}$ & $\begin{array}{l}1.000 \text { [Reference] } \\
0.547(0.061-4.890) \\
1.216(0.167-8.854)\end{array}$ & $\begin{array}{l}0.589 \\
0.847\end{array}$ & $\begin{array}{l}1.000 \text { [Reference] } \\
0.260(0.023-2.900) \\
0.331(0.036-3.040)\end{array}$ & $\begin{array}{l}0.273 \\
0.329\end{array}$ \\
\hline $\begin{array}{l}\text { Tumour histology } \\
\text { IDC } \\
\text { Non-IDC } \\
\end{array}$ & $\begin{array}{l}1.000 \text { [Reference] } \\
1.186 \text { (0.505-2.783) }\end{array}$ & 0.695 & $\begin{array}{l}1.000 \text { [Reference] } \\
0.819(0.308-2.179)\end{array}$ & 0.689 \\
\hline $\begin{array}{l}\text { Surgery } \\
\text { Mastectomy } \\
\text { WLE }\end{array}$ & $\begin{array}{l}1.000 \text { [Reference] } \\
0.316(0.152-0.659)\end{array}$ & 0.002 & $\begin{array}{l}1.000 \text { [Reference] } \\
0.608(0.243-1.522)\end{array}$ & 0.288 \\
\hline $\begin{array}{l}\text { Chemotherapy } \\
\text { Yes } \\
\text { No } \\
\end{array}$ & $\begin{array}{l}1.000 \text { [Reference] } \\
0.844(0.453-1.573)\end{array}$ & 0.593 & $\begin{array}{l}1.000 \text { [Reference] } \\
1.490(0.633-3.507)\end{array}$ & 0.361 \\
\hline $\begin{array}{l}\text { Radiation therapy } \\
\text { Yes } \\
\text { No } \\
\end{array}$ & $\begin{array}{l}1.000[\text { Reference] } \\
0.817(0.463-1.442)\end{array}$ & 0.486 & $\begin{array}{l}1.000 \text { [Reference] } \\
0.934 \text { (0.453-1.923) }\end{array}$ & 0.853 \\
\hline $\begin{array}{l}\text { Recurrence } \\
\text { Yes } \\
\text { No } \\
\end{array}$ & $\begin{array}{l}1.000[\text { Reference] } \\
10.802(5.949-19.613)\end{array}$ & $\mid<0.001$ & $\begin{array}{l}1.000[\text { Reference] } \\
13.113(6.104-28.169)\end{array}$ & $<0.001$ \\
\hline
\end{tabular}


significance (TABLE 3). Correspondingly, 5-year recurrence-free survival was superior in Chinese women compared to Malay and Indian women $(\mathrm{P}=0.031$, HR $2.575,95 \%$ CI 0.165 to 0.915 and $\mathrm{P}=0.034$, HR 2.090, 95\% CI 0.514 to 0.929 respectively), but was similar between Malay and Indian women ( $\mathrm{P}=0.987)$ (FIGURE 1). No significant trend was observed with 5-year overall survival $(\mathrm{P}=0.196)$ (FIGURE
2). We next stratified the women by ethnicity and examined the groups for any significant differences in clinical presentation and tumor features. Chinese women were more often older than Malay and Indian women $(\mathrm{P}<0.001)$ at the time of diagnosis, but apart from this, women from the three major ethnic groups shared similar tumor characteristics (TABLE 4). The stage at diagnosis was relatively similar across

TABLE 3. Univariate and Cox proportional hazards model analyses of recurrence-free survival correlated with clinicopathological parameters and treatments received in patients with non-metastatic disease.

\begin{tabular}{|c|c|c|c|c|}
\hline \multirow[b]{2}{*}{ Parameter } & \multicolumn{2}{|l|}{ Univariate analysis } & \multicolumn{2}{|l|}{ Multivariate analysis } \\
\hline & $\operatorname{HR}(95 \% \mathrm{Cl})$ & $P$ value & $\operatorname{HR}(95 \% \mathrm{Cl})$ & $P$ value \\
\hline Median age (years) & $0.983(0.962-1.004)$ & 0.110 & $0.981(0.956-1.006)$ & 0.128 \\
\hline $\begin{array}{l}\text { Ethnicity } \\
\text { Chinese } \\
\text { Malay } \\
\text { Indians }\end{array}$ & $\begin{array}{l}1.000 \text { [Reference] } \\
2.091(1.064-4.110) \\
2.097(1.041-4.226)\end{array}$ & $\begin{array}{l}0.032 \\
0.038\end{array}$ & $\begin{array}{l}1.000 \text { [Reference] } \\
1.553(0.739-3.263) \\
2.761(1.263-6.039)\end{array}$ & $\begin{array}{l}0.245 \\
0.011\end{array}$ \\
\hline $\begin{array}{l}\text { Disease stage } \\
\text { I } \\
\text { II } \\
\text { III }\end{array}$ & $\begin{array}{l}1.000 \text { [Reference] } \\
2.052(0.926-4.550) \\
6.139(2.812-13.402)\end{array}$ & $\begin{array}{l}0.077 \\
<0.001\end{array}$ & $\begin{array}{l}1.000 \text { [Reference] } \\
1.560(0.663-3.667) \\
5.435(2.110-14.000)\end{array}$ & $\begin{array}{l}0.308 \\
<0.001\end{array}$ \\
\hline \begin{tabular}{|l} 
Tumour grade \\
1 \\
2 \\
3 \\
\end{tabular} & $\begin{array}{l}1.000 \text { [Reference] } \\
0.417(0.084-2.068) \\
0.875(0.213-3.591)\end{array}$ & $\begin{array}{l}0.284 \\
0.853\end{array}$ & $\begin{array}{l}1.000 \text { [Reference] } \\
1.065(0.113-10.081) \\
1.764(0.207-15.055)\end{array}$ & $\begin{array}{l}0.956 \\
0.604\end{array}$ \\
\hline $\begin{array}{l}\text { Tumour histology } \\
\text { IDC } \\
\text { Non-IDC }\end{array}$ & $\begin{array}{l}1.000 \text { [Reference] } \\
1.502(0.742-3.043)\end{array}$ & 0.458 & $\begin{array}{l}1.000[\text { Reference] } \\
1.118(0.479-2.613)\end{array}$ & 0.796 \\
\hline $\begin{array}{l}\text { Surgery } \\
\text { Mastectomy } \\
\text { WLE }\end{array}$ & $\begin{array}{l}1.000 \text { [Reference] } \\
0.458(0.262-0.802)\end{array}$ & 0.006 & $\begin{array}{l}1.000 \text { [Reference] } \\
0.719(0.350-1.478)\end{array}$ & 0.370 \\
\hline $\begin{array}{l}\text { Chemotherapy } \\
\text { Yes } \\
\text { No }\end{array}$ & $\begin{array}{l}1.000 \text { [Reference] } \\
1.153(0.646-2.058)\end{array}$ & 0.631 & $\begin{array}{l}1.000 \text { [Reference] } \\
0.807(0.393-1.657)\end{array}$ & 0.558 \\
\hline $\begin{array}{l}\text { Radiation therapy } \\
\text { Yes } \\
\text { No }\end{array}$ & $\begin{array}{l}1.000 \text { [Reference] } \\
0.989(0.599-1.634)\end{array}$ & 0.967 & $\begin{array}{l}1.000 \text { [Reference] } \\
0.810(0.419-1.566)\end{array}$ & 0.530 \\
\hline
\end{tabular}

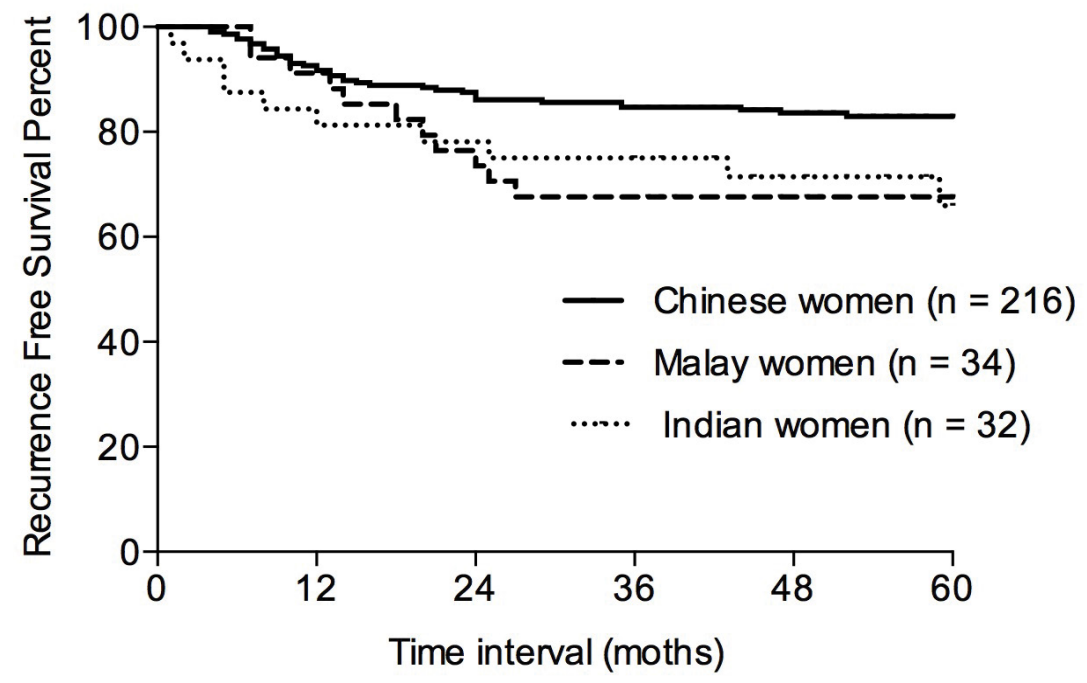

\begin{tabular}{|c|c|c|}
\hline Comparison & Hazard Ratio & 95\% Cl \\
\hline Chinese vs Malay women & 2.575 & $0.165-0.915$ \\
\hline Chinese vs Indian women & 2.090 & 0.031 \\
\hline Malay vs Indian women & 1.012 & 0.034 \\
\hline
\end{tabular}

FIGURE 1. Kaplan-Meier survival analysis of 5-year recurrence-free survival stratified by ethnicity ( $\mathrm{n}=\mathbf{2 8 2}$ ). 


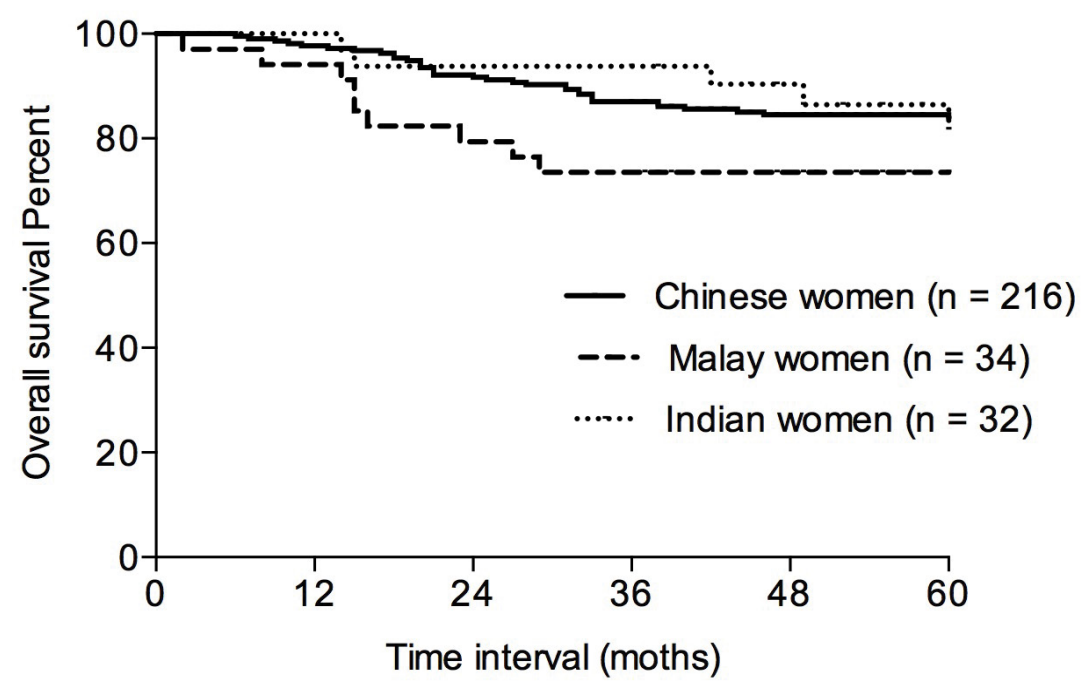

\begin{tabular}{|c|c|c|c|}
\hline Comparison & Hazard Ratio & $\mathbf{9 5 \%} \mathbf{~ C l}$ & P value \\
\hline Chinese vs Malay women & 2.285 & $0.175-1.095$ & 0.077 \\
\hline Chinese vs Indian women & 1.034 & $0.409-2.618$ & 0.943 \\
\hline Malay vs Indian women & 1.909 & $0.665-5.480$ & 0.229 \\
\hline
\end{tabular}

FIGURE 2. Kaplan-Meier survival analysis of 5-year overall survival stratified by ethnicity ( $\mathrm{n}=\mathbf{2 8 2}$ ).

\begin{tabular}{|c|c|c|c|c|}
\hline Parameter & Chinese women $(n=216)$ & Malay women $(n=34)$ & Indian women (n=32) & $P$ value \\
\hline Median age (years) & $56(27-90)$ & $50(33-67)$ & $50(38-79)$ & $<0.001$ \\
\hline $\begin{array}{c}\text { Disease stage } \\
\text { I } \\
\text { III } \\
\text { III }\end{array}$ & $\begin{array}{l}71 \\
97 \\
48\end{array}$ & $\begin{array}{c}6 \\
17 \\
11\end{array}$ & $\begin{array}{c}8 \\
20 \\
4\end{array}$ & 0.118 \\
\hline Median tumour size $(\mathrm{mm})$ & $25(1-119)$ & $30(5-170)$ & $25(3-60)$ & 0.099 \\
\hline $\begin{array}{c}\text { N status } \\
\text { N0 } \\
\text { N1 } \\
\text { N2 } \\
\text { N3 }\end{array}$ & $\begin{array}{l}133 \\
50 \\
12 \\
21\end{array}$ & $\begin{array}{l}16 \\
11 \\
2 \\
5\end{array}$ & $\begin{array}{l}21 \\
7 \\
2 \\
2\end{array}$ & 0.736 \\
\hline $\begin{array}{c}\text { Tumour grade } \\
1 \\
2 \\
3\end{array}$ & $\begin{array}{c}4 \\
41 \\
161\end{array}$ & $\begin{array}{c}0 \\
3 \\
29\end{array}$ & $\begin{array}{c}2 \\
2 \\
27\end{array}$ & 0.088 \\
\hline \begin{tabular}{|c|} 
Tumour histology \\
IDC \\
ILC \\
Metaplastic carcinoma \\
Medullary carcinoma \\
Mucinous carcinoma \\
Papillary carcinoma \\
Tubular carcinoma \\
Invasive carcinoma (not specified)
\end{tabular} & $\begin{array}{c}197 \\
4 \\
5 \\
1 \\
1 \\
2 \\
0 \\
1 \\
6 \\
\end{array}$ & $\begin{array}{l}29 \\
0 \\
2 \\
\\
2 \\
0 \\
0 \\
0 \\
1\end{array}$ & $\begin{array}{l}28 \\
2 \\
1 \\
\\
0 \\
0 \\
0 \\
0 \\
1 \\
\end{array}$ & 0.427 \\
\hline $\begin{array}{c}\text { Surgery } \\
\text { Mastectomy } \\
\text { WE }\end{array}$ & $\begin{array}{l}128 \\
88\end{array}$ & $\begin{array}{l}14 \\
20\end{array}$ & $\begin{array}{l}16 \\
16\end{array}$ & 0.109 \\
\hline $\begin{array}{l}\text { Chemotherapy } \\
\text { Yes } \\
\text { No }\end{array}$ & $\begin{array}{c}148 \\
61\end{array}$ & $\begin{array}{c}28 \\
5 \\
\end{array}$ & $\begin{array}{c}21 \\
9 \\
\end{array}$ & 0.233 \\
\hline $\begin{array}{l}\text { Radiation therapy } \\
\text { Yes } \\
\text { No }\end{array}$ & $\begin{array}{c}118 \\
93 \\
\end{array}$ & $\begin{array}{c}26 \\
7 \\
\end{array}$ & $\begin{array}{l}14 \\
16 \\
\end{array}$ & 0.020 \\
\hline $\begin{array}{l}\text { Recurrence } \\
\text { Yes } \\
\text { No }\end{array}$ & $\begin{array}{r}36 \\
180 \\
\end{array}$ & $\begin{array}{l}11 \\
23 \\
\end{array}$ & $\begin{array}{l}10 \\
22\end{array}$ & 0.027 \\
\hline $\begin{array}{l}\text { Death } \\
\text { Yes } \\
\text { No }\end{array}$ & $\begin{array}{c}35 \\
181\end{array}$ & $\begin{array}{c}9 \\
25\end{array}$ & $\begin{array}{c}5 \\
27\end{array}$ & 0.327 \\
\hline
\end{tabular}




\begin{tabular}{|l|l|l|l|l|}
\hline TABLE 5. Logistic regression of clinicopathological parameters and radiation treatment $(\mathbf{n}=\mathbf{2 3 3})$. \\
\hline Parameter & Odds Ratio & Standard Error & P value & $95 \%$ \\
\hline Ethnicity & 1.439 & 0.785 & 0.504 & $0.494-4.190$ \\
\hline Age & 0.998 & 0.014 & 0.906 & $0.971-1.027$ \\
\hline Surgery type ${ }^{\dagger}$ & 18.345 & 7.947 & $<0.001$ & $7.848-42.882$ \\
\hline Tumour size & 1.011 & 0.009 & 0.211 & $0.994-1.028$ \\
\hline Nodal involvement & 7.326 & 2.896 & $<0.001$ & $3.375-15.900$ \\
\hline${ }^{*}$ Chinese vs Malay; ${ }^{+}$Mastectomy vs Wide Local Excision
\end{tabular}

the 3 groups, with more than half of all the women presenting with Stage II disease. More Malay women $(58.8 \%)$ were treated with WLE than with mastectomy and this was the reason for Malay women being more likely to receive radiation treatment $(\mathrm{P}=0.020)$. This association was further confirmed on multivariate analysis which showed that the likelihood of receiving radiation was dependent on the type of surgery $(\mathrm{P}<0.001$, OR 18.345, 95\% CI 7.848 to 42.882) and extent of nodal disease $(\mathrm{P}<0.001$, OR $7.326,95 \%$ CI 3.375 to 15.900 ) (TABLE 5). No association with ethnicity was observed $(\mathrm{P}=0.504)$.

\section{Discussion}

Higher rates of disease recurrence, particularly that of systemic recurrence, is seen in women with TNBC and account for the poor prognosis associated with this subtype $[3,9]$. Disease recurrence, which occurred in $20 \%$ of the women in our study, was associated with poor overall survival and was predicted by disease stage at presentation and by ethnicity. Among the three major ethnic groups, Chinese women with TNBC were the least likely to relapse and consequently, 5-year recurrencefree survival was longer among Chinese women compared to Malay and Indian women.

Risk of recurrence correlated strongly with the stage at presentation and showed an incremental increase with the stage. Compared to women with Stage I disease, women with Stage II cancers had a 1.5-fold higher risk of recurrence and women with Stage III cancers had a 5-fold higher risk. The majority of recurrences were systemic in nature and tended to occur within the first 5 years of diagnosis, consistent with the known behavior of TNBC [3]. Even among women with an increased risk of local failure from defaulting on whole breast radiation after WLE, disease recurrence was more often systemic, without locoregional recurrence. Treatment, in terms of chemotherapy, radiation and the surgical approach, showed no significant association with the risk of recurrence. The apparent survival benefit observed with WLE was a result of these women having smaller and less advanced tumors and was an expected consequence given that tumor size is one of the main eligibility criteria for WLE. Some studies have found survival outcomes to be superior in women treated with breast conservation compared to those treated with mastectomy [10]. Our previous work, however, suggests that the surgical approach does not affect survival. Poorer outcomes among women who undergo a mastectomy can be explained by the tendency for more elderly women and for those with more severe co-morbidities to be treated with mastectomy. Clinicians, and often the women themselves, are more inclined towards mastectomy so as to avoid the morbidity associated with a repeat operation should the initial margins be inadequate and with postoperative radiation. Survival was equivalent after adjusting for age [11]. Similarly, we also reported that breast conservation was not associated with poorer survival in women with TNBC [12].

Despite the propensity for early systemic recurrence, $80 \%$ of the women in our study remained free of disease and are expected to have a good long-term outcome. Recurrence risk in TNBC declines sharply after the initial few years and continues to decline thereafter, such that the risk beyond 5 years falls bellows that of the more favorable ER-positive tumors $[1,3]$. We made an interesting observation that apart from disease stage at presentation, ethnicity was also an independent predictor of disease recurrence. Outcomes appeared more favorable in Chinese women. Disease recurrence developed in $17 \%$ of the Chinese women, but in more than $30 \%$ of the Malay and Indian women in our study. Correspondingly, recurrence-free survival was poorer among Malay and Indian women compared to Chinese women and 
was not related to the stage at presentation. Although more Malay women were diagnosed with Stage III disease, an observation that we had reported in previous studies, this trend was not statistically significant across the three major ethnic groups $[13,14]$. Overall, more than twothirds of the women had presented with early Stage I and II disease. Tumour grade, size, and nodal burden were also not significantly different across the ethnic groups, and as such, the superior outcomes in Chinese women cannot be explained by them presenting earlier and with more favorable tumor characteristics.

Ethnic variation in survival has been previously reported and breast cancer-specific mortality is disproportionately higher among African American women [15-18].The socioeconomic disparity has often been cited as a major contributor, with women in lower socioeconomic groups having limited access to screening, early detection, and treatments $[19,20]$. Attitudes and compliance to treatments may also differ and furthermore, poor risk factors, such as obesity, poor nutrition, and comorbidities, tend to be more common in those from low socioeconomic groups [21]. These can have a direct biological effect on tumor response and can also influence treatment preferences and tolerability [22-26]. Socioeconomic factors are unlikely to be relevant in our local context where the local healthcare setup accords easily accessible and affordable healthcare to all, with government subsidies being made available to those with insufficient financial means. Instead, the disparity in outcomes could be because certain ethnic groups may have a disproportionately higher prevalence of inherently aggressive tumors, as evidenced by published data reporting ethnic survival disparities even after adjusting for socioeconomic factors and treatment variability [15,18,27,28]. African American women are particularly predisposed to TNBC and a higher incidence of node-positive $\mathrm{T} 1$ tumors have been observed, raising the possibility that TNBCs arising in African American women may have a predilection for early nodal spread [2932]. Such ethnic variation may have a genetic basis and may not be modifiable by changes in environment and lifestyle. Epidemiological studies have reported that the prevalence of TNBC in African American women was significantly lower than that in native African women in Ghana, but it was still more than 3 -folder higher than white non-Hispanic women $[33,34]$.Little is known about the prevalence of TNBC among Asians. Epidemiological studies have included Asians, but the women categorized as 'Asians' in the Surveillance, Epidemiology and End Results Program (SEER) registry comprise very diverse groups of people and no distinction is made between new migrants and second- or third-generation Asians [32]. We found TNBC more common among Indian and Malay women compared to Chinese women, but this needs further validation in larger cohort studies. Our small study numbers reflect the difficulty in studying TNBC, given that it comprises only $10 \%$ to $15 \%$ of all breast cancers. However, our study captures a good proportion of Singaporean women diagnosed with TNBC and since both our institutes follow similar diagnostic and management protocols, outcomes are comparable.

Gene expression profiling has identified six distinct molecular subtypes among TNBCs, each associated with different clinical outcomes [5]. Prognosis is generally better with the immunomodulatory IM and basal (BL1 and BL2) subtypes compared to the mesenchymal $M$ and luminal androgen receptor LAR subtypes $[29,35,6]$ and could be a result of the different pathways activated in each subtype. For instance, tumors of the basal subtype (BL1 and BL2) are enriched in genes modulating cell cycle and DNA damage response, rendering them highly chemo-sensitive and account for the better outcomes seen with this subtype [37]. The observed ethnic variation in survival among women with TNBC raises the possibility that certain TNBC subtypes may be more prevalent among certain ethnic groups. Ethnic variation in molecular subtypes has been reported, [32] and it is possible that Chinese women tend to have more favorable TNBC subtypes. This warrants further evaluation as ethnicity could then have potential value as a surrogate marker of molecular TNBC subtype. In the current setting where molecular gene profiling remains too costly and impractical for routine clinical use, a predictive model incorporating easily obtainable parameters, such as ethnicity and disease stage at presentation, could better stratify TNBC according to the expected outcome and response to treatment. This would serve to improve prognostication and to better guide 
treatment decision-making.

\section{Conclusion}

In keeping with the published literature, overall survival was poorer among women with TNBC and correlated strongly with disease recurrence. The stage at presentation and ethnicity showed an independent association with disease recurrence. Recurrence-free survival was superior among Chinese women compared to Malay and Indian women, despite the stage at presentation and tumor characteristics were comparable across the ethnic groups. Radiation treatment was more frequent among Malay women, a consequence of the higher rates of breast conservation, but otherwise, there were no significant differences in the types of treatment received.

\section{Acknowledgments}

This work was supported in part by the National Medical Research Council IRG grant (NMRC/CIRG/1464/2016).

\section{Conflict of Interest Statement}

All authors declare no conflict of interest. 


\section{References}

R Dent, M Trudeau, KI Pritchard, et al. Triple-negative breast cancer: clinical features and patterns of recurrence. Clin Cancer Res. 13(15 Pt 1), 4429-4434 (2007).

BG Haffty, Q Yang, M Reiss, et al. Locoregional relapse and distant metastasis in conservatively managed triple negative early-stage breast cancer. J Clin Oncol. 24(36), 5652-5657 (2006).

WD Foulkes, IE Smith, JS Reis-Filho. Triple-negative breast cancer. $N$ Engl J Med. 363(20), 1938-1948 (2010).

M Tischkowitz, JS Brunet, LR Begin, et al. Use of immunohistochemical markers can refine prognosis in triple negative breast cancer. BMC Cancer. 7134 (2007).

BD Lehmann, JA Bauer, X Chen, et al. Identification of human triple-negative breast cancer subtypes and preclinical models for selection of targeted therapies. J Clin Invest. 121(7), 2750-2767 (2011).

ME Hammond, DF Hayes, M Dowsett, et al. American Society of Clinical Oncology/College of American Pathologists guideline recommendations for immunohistochemical testing of estrogen and progesterone receptors in breast cancer. Arch Pathol Lab Med. 134(6), 907-922 (2010).

AC Wolff, ME Hammond, DG Hicks, et al. Recommendations for a human epidermal growth factor receptor 2 testing in breast cancer: American Society of Clinical Oncology/College of American Pathologists clinical practice guideline update. J Clin Oncol. 31(31), 3997-4013 (2013).

TJ Whelan, JP Pignol, MN Levine, et al. Long-term results of hypofractionated radiation therapy for breast cancer. $N$ Engl J Med. 362(6), 513-520 (2010).

T Sorlie, CM Perou, R Tibshirani, et al. Gene expression patterns of breast carcinomas distinguish tumor subclasses with clinical implications. Proc Natl Acad Sci USA. 98(19), 10869-10874 (2001).

S Agarwal, L Pappas, L Neumayer, et al. Effect of breast conservation therapy vs mastectomy on disease-specific survival for early-stage breast cancer. JAMA Surg. 149(3), 267-274 (2014).

PM Chan, BA Choo, T Zhang, et al. Mastectomy rates remain high in Singapore and are not associated with poorer survival after adjusting for age. Springerplus. 4685 (2015).

FC Adkins, AM Gonzalez-Angulo, $\mathrm{X}$ Lei, et al. Triple-negative breast cancer is not a contraindication for breast conservation. Ann Surg Oncol. 18(11), 3164-3173 (2011).

EY Tan, HB Wong, BK Ang, et al. Locally advanced and metastatic breast cancer in a tertiary hospital. Ann Acad Med Singapore. 34(10), 595-601 (2005).

CH Pek, EY Tan, JJ Chen, et al. Advanced breast cancer-are we doing enough? Breast J. 18(6), 644-646 (2012).

LA Newman, KA Griffith, I Jatoi, et al. Meta-analysis of survival in African American and white American patients with breast cancer: ethnicity compared with socioeconomic status. J Clin Oncol. 24(9), 1342-1349 (2006).

L Holmes, Jr F Opara, J Hossain. A five-year breast cancer-specific survival disadvantage of African American women. Afr J Reprod Health. 14(3), 195-200 (2010).

SM Conroy, G Maskarinec, LR Wilkens, et al. Obesity and breast cancer survival in ethnically diverse postmenopausal women: the Multiethnic Cohort Study. Breast Cancer Res Treat. 129(2), 565-574 (2011).

CM Tammemagi, D Nerenz, C Neslund-Dudas, et al. Comorbidity and survival disparities among black and white patients with breast cancer. JAMA. 294(14), 1765-1772 (2005).

SS Gorin, JE Heck, B Cheng, et al. Delays in breast cancer diagnosis and treatment by racial/ethnic group. Arch Intern Med. 166(20), 2244-2252 (2006).

CI Li, KE Malone, JR Daling. Differences in breast cancer stage, treatment, and survival by race and ethnicity. Arch Intern Med. 163(1), 49-56 (2003).

L Vona-Davis, DP Rose. The influence of socioeconomic disparities on breast cancer tumor biology and prognosis: a review. J Womens Health (Larchmt). 18(6), 883-893 (2009).

RE Patterson, LA Cadmus, JA Emond, et al. Physical activity, diet, adiposity and female breast cancer prognosis: a review of the epidemiologic literature. Maturitas. 66(1), 5-15 (2010).

EE Calle, R Kaaks. Overweight, obesity and cancer: epidemiological evidence and proposed mechanisms. Nat Rev Cancer. 4(8), 579-591 (2004).

JJ Griggs, ME Sorbero, GH Lyman. Undertreatment of obese women receiving breast cancer chemotherapy. Arch Intern Med. 165(11), 1267-1273 (2005).

M Barbaric, E Brooks, L Moore, et al. Effects of physical activity on cancer survival: a systematic review. Physiother Can. 62(1), 25-34 (2010).

TP Srokowski, $S$ Fang, GN Hortobagyi, et al. Impact of diabetes mellitus on complications and outcomes of adjuvant chemotherapy in older patients with breast cancer. J Clin Oncol. 27(13), 2170-2176 (2009).

S Harper, J Lynch, SC Meersman, et al. Trends in area-socioeconomic and race-ethnic disparities in breast cancer incidence, stage at diagnosis, screening, mortality, and survival among women ages 50 years and over (1987-2005). Cancer Epidemiol Biomarkers Prev. 18(1), 121131 (2009).

KS Albain, JM Unger, JJ Crowley, et al. Racial disparities in cancer survival among randomized clinical trials patients of the Southwest Oncology Group. J Natl Cancer Inst. 101(14), 984-992 (2009).

J Iqbal, O Ginsburg, PA Rochon, et al. Differences in breast cancer stage at diagnosis and cancer-specific survival by race and ethnicity in the United States. JAMA. 313(2), 165-173 (2015).

LA Stead, TL Lash, JE Sobieraj, et al. Triple-negative breast cancers are increased in black women regardless of age or body mass index. Breast Cancer Res. 11(2), R18 (2009).

KR Bauer, M Brown, RD Cress, et al. Descriptive analysis of estrogen receptor (ER)-negative, progesterone receptor (PR)-negative, and HER2-negative invasive breast cancer, the so-called triplenegative phenotype: a population-based study from the California Cancer Registry. Cancer. 109(9), 1721-1728 (2007).

AW Kurian, K Fish, SJ Shema, et al. Lifetime risks of specific breast cancer subtypes among women in four racial/ ethnic groups. Breast Cancer Res. 12(6), R99 (2010). 
D Huo, F Ikpatt, A Khramtsov, et al. Population differences in breast cancer: a survey in indigenous African women reveals over-representation of triplenegative breast cancer. $J$ Clin Oncol. 27(27), 4515-4521 (2009).

A Stark, CG Kleer, I Martin, et al. African ancestry and higher prevalence of triple-negative breast cancer: findings from an international study. Cancer. 116(21), 4926-4932 (2010).

AE Teschendorff, A Miremadi, SE Pinder, et al. An immune response gene expression module identifies a good prognosis subtype in estrogen receptor negative breast cancer. Genome Biol. 8(8), R157 (2007).

AM Gonzalez-Angulo, K Stemke-
Hale, SL Palla, et al. Androgen receptor levels and association with PIK3CA mutations and prognosis in breast cancer. Clin Cancer Res. 15(7), 2472-2478 (2009).

IA Mayer, VG Abramson, BD Lehmann, et al. New strategies for triplenegative breast cancer-deciphering the heterogeneity. Clin Cancer Res. 20(4), 782-790 (2014). 